\title{
Generalização de Mandos Aprendidos pelo PECS (Picture Exchange Communication System) em Crianças com Transtorno do Espectro Autista
}

\author{
Juliana Campos de Jesus \\ Thais Porlan Oliveira ${ }^{1}$ \\ Junio Vieira de Rezende \\ Universidade Federal de Minas Gerais, Belo Horizonte, MG, Brasil \\ Instituto Nacional de Ciência e Tecnologia sobre Comportamento, \\ Cognição e Ensino - INCT/ECCE, São Carlos, SP, Brasil
}

\begin{abstract}
Resumo
O ensino de comportamento verbal com o uso de comunicação alternativa é um instrumento para melhorar a interação social de crianças com Transtorno do Espectro Autista (TEA). O objetivo do estudo foi ensinar mandos com o Picture Exchange Communication System (PECS) e avaliar a sua generalização entre ambientes. Os mandos treinados consistem na troca de cartões com estímulos visuais pelos próprios itens. Participaram do estudo quatro crianças diagnosticadas com TEA, com idade entre 6 e 12 anos. Após treino do PECS, professora e mães receberam instruções e registraram a emissão de mandos (teste de generalização) nos ambientes escolar e domiciliar. Os resultados mostraram que para três crianças o ensino de mandos foi bem sucedido e que ocorreu a generalização para outros itens nos dois contextos avaliados. É discutida a necessidade de maior controle de variáveis que interfiram no treino para a aquisição de mandos, além de planejamento da generalização pelas crianças para ambientes, pessoas e itens diferentes.
\end{abstract}

Palavras-chave: Transtorno do Espectro Autista, comportamento verbal, mando, PECS, generalização.

\section{Generalization of Mands Learned by PECS (Picture Exchange Communication System) in Children with Autism}

\begin{abstract}
Verbal training with the use of alternative communication is a tool for improving the social interaction of children with Autism Spectrum Disorder (TEA). The aim of this study was to promote generalization of trained mands through the Picture Exchange Communication System (PECS). Trained mands comprise the exchange of pictures with visual stimuli by the items themselves. There were 4 autists aged between 6 and 12 years old, who took part in the study. After the PECS training, the teacher and the mothers were instructed to take notes of the mands performed in school and home settings. Results
\end{abstract}

Endereço para correspondência: Universidade Federal de Minas Gerais, Faculdade de Filosofia e Ciências Humanas, Departamento de Psicologia, Laboratório da Análise do Comportamento, Av. Antônio Carlos, 6627, Pampulha, Belo Horizonte, MG, Brasil 31270-910 - E-mail: thais.porlan@gmail.com

Financiamento: Conselho Nacional de Desenvolvimento Científico e Tecnológico (CNPq) -processo\#573972/2008-7 e Fundação de Amparo à Pesquisa do Estado de São Paulo (FAPESP) processo\#08/57705-8. 
have shown that 3 children met the learning criterion for PECS and that there was a generalization for other settings. The need for greater control of the variables that interfere with mand acquisitions and the children planning for generalization of environment, people and different items have been discussed.

Keywords: Autism, verbal behavior, mand, PECS, generalization.

\section{Generalización de Mandos Aprendidos por PECS (Picture Exchange Communication System) en los Niños Autistas}

\section{Resumen}

Entrenamiento verbal con el uso de la comunicación alternativa es una herramienta para mejorar la interacción social de los niños con trastorno del espectro autista (TEA). El objetivo era enseñar a los comandos con el Sistema Picture Exchange Communication (PECS) y evaluar su generalización. Mandos entrenados consisten en el intercambio de cartas con los estímulos visuales por elementos propios. Los participantes del estudio fueron cuatro niños diagnosticados con TEA, de 6 a 12 años. Después del entrenamiento del PECS, profesor y madres fueron instruidos y grabó las exigencias de emisiones (prueba de generalización) en el entorno escolar y el hogar. Los resultados mostraron que a tres hijos demandas del entrenamiento fue un éxito y lo que pasó generalización a otros artículos en los dos contextos evaluados. Se discutió la necesidad de mayores variables de control que interfieren con lo entrenamiento para adquirir comandos y la generalización de la planificación para los niños a los ambientes, personas y diferentes elementos.

Palabras clave: Autismo, conducta verbal, mando, PECS, generalización.

O repertório verbal tem como uma de suas funções mais importantes demonstrar ao outro nossas necessidades, desde as mais básicas, como sentir sede ou fome, até as mais complexas, como expressar um sentimento. O mando é definido como uma resposta verbal que especifica necessidades da pessoa e, portanto, é um operante verbal que tem por característica o benefício direto do falante. Independente da topografia do mando (seja um pedido, o gesto de apontar objetos, figuras ou a troca de figuras), ele é definido por sua função, determinada pelas condições antecedentes aversivas ou de privação e pela oportunidade de produzir a consequência especificada por ele (Skinner, 1957). Assim, é um exemplo de mando uma criança dizer "estou com fome" e a mãe lhe oferecer alimento. As condições de estimulação aversiva e a privação antecedentes à emissão de um mando são denominadas operações motivacionais que modificam a efetividade do reforçador ou punidor e alteraram a classe de comportamentos ligadas àquelas consequências (Laraway, Snycerski, Michael, \& Poling, 2003).
O mando, portanto, possibilita maior controle socialmente mediado sobre o ambiente, permitindo que a pessoa se relacione de forma mais adaptativa ao meio (Murphy, Barnes-Holmes, \& Barnes-Holmes, 2005; Vollmer et al., 2007). Crianças com déficits no repertório comunicativo como nos casos de TEA, apresentam limitações nas oportunidades de interação social, gerando dificuldades para que tenham acesso às suas necessidades mais básicas, como por exemplo, demonstrar para o ouvinte se ela tem sede ou o que prefere comer (Murphy et al., 2005).

O Transtorno do Espectro Autista (TEA) abrange quadros situados num contínuo de comprometimento das capacidades de interação social e comunicação (American Psychiatric Association, 2013). Existem dois grandes critérios diagnósticos para o TEA: (a) déficits em comunicação e interação social e (b) comportamentos, interesses e atividades repetitivas e restritas. A categoria comunicação e interação social agrupam as dificuldades em relação à comunicação verbal (vocal e não vocal), falta de reciprocidade 
social e incapacidade de desenvolver e manter com seus pares relacionamentos apropriados ao seu nível de desenvolvimento (American Psychiatric Association, 2013).

Nos casos em que os indivíduos desenvolvem a fala, ela pode ser imatura, ecolálica, apresentar prosódia anormal ou reversão de pronomes (Bosa \& Schmidt, 2003; Gadia, Tuchman, \& Rotta, 2004; Martins, 2001; Sundberg \& Partington, 1998). Devido à alta prevalência de casos que não se comunicam por meio da linguagem oral, as intervenções com foco na aquisição de uma comunicação funcional, com o uso da chamada Comunicação Suplementar e/ou Alternativa (CSA), podem ser um meio para promover a comunicação de crianças com TEA (Ganz \& Simpson, 2004; Lampreia, 2004; Llaneza et al., 2010; Martins, 2001). Dentre os métodos de intervenção em CSA, o Picture Exchange Communication System (PECS) é um protocolo de ensino no qual a criança aprende a trocar estímulos visuais (figuras) por objetos ou atividades de interesse (Bondy \& Frost, 1994). A primeira habilidade ensinada de acordo com o protocolo do PECS é o mando, ou seja, as crianças aprendem a conseguir a atenção de outras pessoas e pedir pelo que desejam com auxílio das figuras (Ganz, Simpson, \& Corbin-Newsome, 2008; Jurgens, Anderson, \& Moore, 2009; Tien, 2008).

Intervenções direcionadas aos problemas de comunicação no TEA encontram como obstáculo adicional a dificuldade observada nessas crianças em generalizar um comportamento aprendido em um ambiente para outros contextos (Capellari, 2010; Fazzio, 2002; Fragale et al., 2012; Green, 2011; Klin, Chawarska, Rubin, \& Volkmar, 2006). Ao aprenderem a emitir mandos, crianças com TEA podem falhar na sua utilização fora do ambiente no qual foram treinados, quando na presença de pessoas diferentes de quem os ensinou ou diante de estímulos diferentes daqueles com os quais receberam o treino (Fragale et al., 2012). Vários autores consideram fundamental que a generalização dos comportamentos adquiridos seja planejada, por exemplo, por meio da repetição da aplicação do procedimento em outros ambientes para os quais se deseja que o repertório adquirido se estenda (Cor- sello, 2005; Fazzio, 2002; Green, 2011; Klin \& Mercadante, 2006; Souza \& Juliani, 2012).

Alguns autores, como Carré, Le Grice, Blampied e Walker (2009), consideraram a importância de estudos sobre as variáveis que facilitam a generalização, como o uso de mais estímulos, diferentes settings e diversos parceiros de comunicação. Neste estudo eles ensinaram as três primeiras fases do PECS a três crianças e avaliaram a generalização dos mandos. O ensino iniciou-se numa sala separada de Educação Especial, gradualmente foi transposto para a sala de aula comum e todas as crianças aprenderam a emitir mandos. Nos testes de generalização realizados em casa e na escola todas as crianças apresentaram baixos níveis de iniciações espontâneas de troca de figuras e apresentaram mais mandos na escola do que em casa.

Dogoe, Banda e Lock (2010) avaliaram a aquisição e generalização de mandos com o uso do PECS em três crianças autistas, modificando os parceiros de comunicação, os ambientes e os estímulos para avaliar generalização. Todos os três participantes adquiram a habilidade e generalizaram para outros ambientes e outros parceiros, mas somente dois participantes apresentaram generalização com estímulos diferentes dos itens que foram treinados. Os autores indicaram a necessidade de mais estudos sobre generalização da habilidade do PECS para novos estímulos, já que a literatura tem focado as condições de generalização para pessoas e ambientes.

Ali, MacFarland e Umbreit (2011) também mostraram resultados de que, embora todos os participantes tenham aprendido a requisitar utilizando figuras, algumas limitações foram verificadas em relação à avaliação da generalização, tais como: a generalização avaliada somente na sala de aula e os participantes requisitaram apenas após o item ter sido oferecido a eles, não realizando iniciações independentes. A aquisição e a generalização de mandos por meio do PECS deste estudo foi adaptada para quatro participantes de sete a quatorze anos, todos com TEA e deficiência visual. Os participantes foram submetidos ao ensino das três primeiras fases do PECS, realizado em uma sala extra, e a generalização avaliada dentro da sala de aula. 
De forma geral, os efeitos de generalização têm sido mensurados durante ou entre a realização das fases de treino (Fidalgo \& Godoi, 2008; Jurgens et al., 2009) com uma quantidade pequena de estímulos ou com os mesmos estímulos utilizados nos treinos (Dogoe et al., 2010) e com pouca variação de pessoas e ambientes (Charlop-Christy, Carpenter, Le, Leblanc, \& Kellet, 2002; Fidalgo \& Godoi, 2008). Assim, a avaliação da generalização de mandos ainda necessita de estudos que propiciem maior diferença entre a situação de ensino e as condições de generalização, para que possa ser verificado com maior clareza se a dificuldade em generalizar que os autistas apresentam pode ser reduzida ou mesmo suprimida se fornecermos condições direcionadas especificamente para propiciar a generalização após o treino.

O objetivo do presente estudo foi avaliar os efeitos do programa interventivo em comunicação alternativa (PECS) na generalização de respostas de mando de quatro crianças com TEA. A generalização dos mandos foi medida em dois ambientes naturais das crianças: a escola e a residência.

\section{Método}

\section{Participantes e Instrumentos}

Participaram do estudo quatro crianças $(\mathrm{P} 1$, P2, P3 e P4) com idades entre 6 e 12 anos, duas meninas e dois meninos diagnosticados com TEA de acordo com o DSM-V. Os critérios de participação foram baixo ou nenhum repertório verbal oral e ausência de exposição prévia ao PECS. Todas as providências éticas neste trabalho seguiram as normas do Comitê de Ética em Pesquisa com Seres Humanos da Universidade Federal de Minas Gerais (UFMG) - Comitê de Ética em Pesquisa - COEP (Parecer $n^{\circ}$ 0662.0.203.000-11).

No início do estudo foi realizada uma entrevista com os pais dos participantes na qual foram levantados, além de outros dados de caracterização, os estímulos potencialmente reforçadores, os quais foram posteriormente submetidos à Avaliação de preferência entre múltiplos estímulos (MSWO) de DeLeon e Iwata (1996). O repertório verbal foi avaliado a partir do relato das professoras e via observação direta no ambiente escolar, em três sessões que duraram entre 15 e 30 min para cada participante, e registrado por meio do inventário Assessment of Basic Language and Learning Skills - ABLLS (Sundberg \& Partington, 1998). Dentre as 544 habilidades que compõem o ABLLS, divididas em 25 áreas específicas, foram considerados os itens relacionados à: comportamento verbal (imitação vocal, tatos e mando), contato visual, imitação motora e habilidade de pareamento com o modelo.

Foi também utilizada a escala Childhood Autism Rating Scale - CARS (Schopler, Reichler, \& Renner, 1988) para avaliação da gravidade do autismo. As informações utilizadas para a avaliação foram obtidas por observação direta e por dados informados pelos pais e professores. Os resultados obtidos nas avaliações estão apresentados na Tabela 1.

P1 foi diagnosticado com um ano e três meses de idade. Apresenta ecolalia, fala na primeira pessoa, pouco contato visual, emite tatos de palavras simples e obedece ordens simples. P2 foi diagnosticada aos quatro anos, apresenta ecolalia imediata e tardia e se comunica muito pouco verbalmente. Em geral, leva as pessoas até o item que deseja ou se aproxima dele, sem fazer contato visual. P3 teve o diagnóstico aos três anos; não verbaliza, apenas emite sons repetitivos, porém sem imitação. Guia as pessoas até seus itens de interesse. Apresenta estereotipias como balanceio de corpo e tamborilar os dedos em objetos. Mantém pouco contato visual e tem dificuldades em seguir regras e permanecer sentada para a realização de atividades cotidianas. P4 recebeu o diagnóstico aos três anos. Emite diversos sons sem função comunicativa. Guia as pessoas até os objetos de interesse, mantendo contato visual com elas.

\section{Materiais e Ambientes}

Foram utilizados estímulos potencialmente reforçadores e coerentes com a rotina da sala de aula, tais como brinquedos, materiais escolares, utensílios domésticos e alimentos. Para P3 e P4, estímulos ausentes na avaliação de preferência foram adicionados nas fases iniciais de treino 
Tabela 1

Caracterização dos Participantes de acordo com Diagnóstico, Sexo, Idade Cronológica, Avaliação CARS e Avaliação ABLLS

\begin{tabular}{lcccc}
\hline \multicolumn{1}{c}{ Características } & P1 & P2 & P3 & P4 \\
\hline Diagnóstico & TEA & TEA & TEA & TEA \\
Sexo & M & F & F & M \\
Idade (anos-meses) & $8-4$ & $11-4$ & $8-8$ & $6-1$ \\
CARS (15-60) & Moderado (31) & Severo (38) & Severo (43.5) & Severo (40.5) \\
ABLLS (\% de itens pontuados) & & & & \\
$\quad$ Imitação vocal & 86,10 & 86,10 & 0,00 & 0,00 \\
$\quad$ Mandos & 35,29 & 11,76 & 4,41 & 2,94 \\
$\quad$ Tatos & 39,72 & 21,91 & 0,00 & 0,00 \\
\hline
\end{tabular}

Nota. CARS $=$ Childhood Autism Rating Scale; ABLLS = Assessment of Basic Language and Learning Skills.

por terem sido observados pelos experimentadores como prováveis reforçadores.

Para a implementação do PECS, foram utilizadas figuras coloridas, medindo $12 \mathrm{~cm}$ por $12 \mathrm{~cm}$ ou $7 \mathrm{~cm}$ por $7 \mathrm{~cm}$, impressas em papel comum e plastificadas, representando estímulos avaliados como reforçadores e estímulos neutros. As imagens correspondiam aos estímulos reais e foram retiradas do software Boardmaker (Sign Language Symbols - PCS) e de sítios virtuais diversos. As figuras eram fixadas com Velcro ${ }^{\circledR}$ em tablados na cor preta, medindo $24 \mathrm{~cm}$ por $30 \mathrm{~cm}$.

Nas fases de treino do PECS, as sessões foram realizadas na escola das crianças, duas ou três vezes por semana, no horário regular em salas de atendimento individual medindo cerca de $12 \mathrm{~m}^{2}$, equipadas com uma mesa infantil de madeira, três cadeiras de tamanho infantil e uma mesa grande de trabalho, onde era posicionada a câmera. Na fase de generalização, as sessões foram realizadas (a) na sala de aula, com a presença adicional da professora e de cerca de seis alunos e (b) em ambiente domiciliar, com a presença da mãe e demais pessoas do convívio familiar e nenhum experimentador.

\section{Delineamento}

O estudo teve como delineamento geral o delineamento do sujeito único, no qual uma mesma variável (treino do PECS) foi introduzida para um mesmo comportamento (respostas de mando), porém em sujeitos diferentes e comparando e compartilhando condições ambientais semelhantes. O delineamento constou de linha de base, fase de intervenção (treino de mandos pelo PECS) e o teste da generalização em dois ambientes, escola e residência.

\section{Procedimento}

No estudo foram manipuladas as variáveis intervalo de tempo entre treino e teste, quantidade de estímulos e variação de ambiente. $\mathrm{Na}$ escola, a generalização de mandos com o uso do PECS foi avaliada com figuras novas, diferentes daquelas treinadas, e com a mesma parceira de comunicação (a professora). Na residência, os mandos foram diferentes do treino tanto com estímulos novos quanto com uma nova parceira de comunicação (a mãe).

\section{Linha de Base e Avaliação de Preferência}

Foram realizadas três sessões de linha de base nas quais o participante, enquanto realizava uma atividade de sua rotina, tinha um objeto de seu interesse presente em seu campo de visão, mas fora de seu alcance, e o tablado à sua disposição, contendo uma figura. Caso o participante pegasse a figura e a entregasse ao experimentador, o item solicitado the seria entregue e regis- 
trar-se-ia que a criança utilizava a troca de figuras como método comunicativo. Após a linha de base, cada participante passou pela avaliação de preferência por reforçadores, realizada em dois dias (DeLeon \& Iwata, 1996).

\section{Ensino do PECS}

Foi realizado o treino das três primeiras fases do PECS: (a) ensino de troca física, (b) ensino da espontaneidade e (c) ensino da discriminação entre estímulos diversos (Bondy \& Frost, 1994). Em cada sessão era realizado um bloco de ensino que continha entre cinco e 20 tentativas. $\mathrm{O}$ número de blocos de tentativas dependia do desempenho de cada participante. O critério para completar cada fase era a realização, de forma independente e correta, de $80 \%$ das tentativas por três sessões consecutivas. As sessões duraram cerca de $15 \mathrm{~min}$, sempre com a presença de três experimentadores: um que realizava a filmagem e os outros dois que interagiam com o participante. Em caso de demonstrações de aversão ou cansaço pelo participante as sessões eram interrompidas

$\mathrm{Na}$ Fase 1 foi treinado o comportamento de trocar a figura, composto pelas respostas de pegar a figura sobre a mesa, estender o braço em direção ao experimentador e colocar a figura na mão do experimentador. Foram utilizados três níveis de ajuda, oferecidos para qualquer uma das três respostas: ajuda total, quando todo o movimento era realizado com o auxílio físico do experimentador; ajuda parcial, quando somente o início do movimento de qualquer das três respostas era direcionado; e ajuda gestual, quando o experimentador indicava com gestos a resposta a ser emitida.

Na Fase 2 foi treinada espontaneidade, ainda com a presença de apenas um estímulo, como na fase anterior. Essa fase foi dividida em três etapas, com níveis crescentes de complexidade do comportamento alvo. Eram oferecidos os três níveis de ajuda presentes na Fase 1. A primeira etapa consistia na troca física da figura, a partir desse momento fixadas com Velcro ${ }^{\circledR}$ ao tablado. $\mathrm{Na}$ segunda etapa, a criança permaneceu próxima ao tablado, mas para realizar a troca deveria levar a figura até o experimentador, que ficava afastado com o item correspondente à figura. $\mathrm{Na}$ terceira etapa, tanto o tablado quanto o experimentador ficavam distantes (aproximadamente de 3 a 5 passos) da criança.

No início da Fase 3 eram dispostas no tablado as figuras correspondentes a um estímulo reforçador e um estímulo neutro. Em seguida, eram dispostas três figuras e a criança deveria escolher a figura de um item em meio a duas outras (distratores), que eram modificadas ao longo das sessões. O número de figuras de estímulos neutros e reforçadores foi gradativamente aumentado até que estivessem disponibilizados vários estímulos neutros e reforçadores ao mesmo tempo, até atingir o numero máximo de cinco estímulos de cada. Outras diferenças desta fase foram a diminuição do tamanho das figuras (que passou de $12 \mathrm{~cm}$ por $12 \mathrm{~cm}$ para de $7 \mathrm{~cm}$ por $7 \mathrm{~cm}$ ) e a retirada de todos os tipos de ajuda presente nas fases anteriores. Ao invés disso, em caso de erro na resposta de discriminação esperada, foram utilizados procedimentos corretivos como a introdução de figuras brancas em lugar dos estímulos neutros, o aumento do número de figuras neutras e a remoção completa dos distratores e uso de ajuda gestual.

Outro procedimento corretivo utilizado na Fase 3 foi a checagem de correspondência (Frost \& Bondy, 1994), necessário para P2. Nesse procedimento, três figuras eram apresentadas enquanto os itens correspondentes permaneciam sobre a mesa. Ao escolher a figura, o participante era solicitado a pegar o item. Tal procedimento visa garantir a discriminação entre estímulos neutros e reforçadores e foi atestada com três acertos consecutivos.

Após o ensino das três primeiras fases do PECS, foi realizada uma extensão da Fase 3, adaptada para este estudo, denominada Fase 3+. A diferença residia na realização do treino na sala de aula, para que as crianças emitissem mandos por itens necessários para completar uma atividade escolar, utilizando estímulos como lápis, cola, tesoura, bola, ou lanche, de acordo com a atividade.

Esta fase foi adaptada e estendida para o ambiente natural para que as crianças emitissem mandos por itens necessários para completar 
uma cadeia de comportamento. Uma operação motivacional foi criada para que mandos fossem emitidos num procedimento de apresentação de três tarefas que as crianças tinham em seu repertório, escolhidas entre os seguintes comportamentos: colorir um desenho, jogar bola, recortar, colar figuras e lanchar (Lechago, Carr, Grow, Love, \& Almason, 2010). A etapa 3+ foi realizada somente para $\mathrm{P} 1, \mathrm{P} 2$ e $\mathrm{P} 4$, pois $\mathrm{P} 3$ não obteve desempenho anterior suficiente. Para P4 foi utilizada somente uma cadeia de comportamento - pedir lanche - na qual o item pedido era comestível, pois o participante não apresentava habilidades escolares em seu repertório e as habilidades de brincar não pareciam suficientemente motivadoras.

\section{Avaliação da Generalização}

Após o ensino do PECS foi realizado um treinamento de quatro horas com as mães e as professoras. Após serem dadas informações acerca das características do TEA e da importância da comunicação, foi apresentado o PECS, seus materiais e processo de ensino. Foram dadas instruções claras sobre como responder adequadamente ao mando das crianças e como criar condições facilitadoras para sua ocorrência.

As mães e as professoras foram instruídas a disponibilizar para os participantes o tablado durante $30 \mathrm{~min}$ por dia, em horários variados, e a registrar o uso do PECS em um formulário específico. $\mathrm{O}$ formulário continha local para registro de: qual o item pedido; local em que ocorreu a troca; quem estava presente e era o ouvinte; liberação/não liberação do item para a criança (e o motivo, caso não tenha sido liberado); presença ou não do item no campo de visão da criança; presença de comportamento vocal no momento da troca e consequências sociais (como elogios) que eventualmente acompanharam a troca. $\mathrm{O}$ tablado de comunicação deveria ficar ao alcance do participante, contendo entre 8 e 10 figuras de itens reforçadores treinados e itens não treinados. Os itens não treinados eram estímulos novos específicos para cada participante, que não haviam sido apresentados no treino e que faziam parte do contexto, como, por exemplo, DVD de filme infantil na residência.
O preenchimento dos formulários ocorreu durante um total de 16 dias, distribuídos em um período de cinco semanas, sendo cinco dias na primeira semana, três dias na segunda semana, nenhum dia na terceira e novamente cinco e três dias nas quarta e quinta semanas, respectivamente. $\mathrm{O}$ mesmo esquema foi aplicado primeiro ao ambiente escolar e depois ao domiciliar, totalizando 10 semanas de registros. A programação inicial foi cumprida com intervalos maiores, devido a imprevistos como faltas à escola e feriados, resultando em um total de três semanas para a avaliação da generalização.

Para cada criança foram confeccionados dois tablados, um para o ambiente escolar e outro para a residência, contendo figuras de itens típicos de cada ambiente. Os itens deveriam permanecer fora do alcance da criança (dentro ou fora do seu campo de visão), não poderiam ser disponibilizados em outras situações e foram sempre oferecidos como consequência aos mandos emitidos por meio do PECS.

Ao final da etapa de preenchimento dos formulários, foi avaliada generalização de respostas de mando em dois contextos diferentes, por parte dos participantes: na sala de aula, onde o comportamento era generalizado para situações diferentes daquelas em que houve treino, sem a presença dos experimentadores, porém na presença da professora que havia participado da Fase 3+; e generalização na residência, na qual o contexto, situação, local e ouvinte foram alterados totalmente.

\section{Concordância entre Observadores}

Todas as sessões de treino foram filmadas e as sessões de generalização foram filmadas parcialmente, uma vez por semana em dias aleatórios. As respostas de mando dos participantes foram confrontadas posteriormente com os registros realizados por um observador neutro a partir da análise de 10 sessões de cada fase e para as sessões de generalização. A concordância entre os observadores foi calculada pela divisão do número de concordâncias pela soma das concordâncias e discordâncias (Ganz et al., 2008). O índice percentual foi obtido multipli- 
cando-se o resultado por 100. A concordância entre observadores obtida foi de $95 \%$.

\section{Resultados}

\section{Ensino}

Na linha de base nenhum dos quatro participantes utilizou as figuras para requisitar pelos itens. Na Tabela 2 estão apresentados os desempenhos dos participantes em todas as fases de ensino do PECS. P3 não alcançou o critério para concluir a fase $2 \mathrm{e}$, portanto, não completou as fases programadas do ensino. Após 69 sessões, o participante demonstrou desinteresse e apresentou comportamentos agressivos ao ser levado à sala, motivo pelo qual foi desligado da pesquisa.

Tabela 2

Total de Sessões (s), Tentativas (t) de Treino e Mandos Treinados (m) por Fase

\begin{tabular}{|c|c|c|c|c|c|c|c|c|c|c|c|c|}
\hline \multirow[b]{2}{*}{ Fases } & \multicolumn{3}{|c|}{$\mathrm{P} 1$} & \multicolumn{3}{|c|}{$\mathrm{P} 2$} & \multicolumn{3}{|c|}{ P3 } & \multicolumn{3}{|c|}{ P4 } \\
\hline & $s$ & $t$ & $m$ & $s$ & $t$ & $m$ & $s$ & $t$ & $m$ & $s$ & $t$ & $m$ \\
\hline Fase 1 & 7 & 35 & & 6 & 30 & & 32 & 160 & & 21 & 105 & \\
\hline Fase 2 & 9 & 45 & & 10 & 50 & & 37 & 185 & & 19 & 95 & \\
\hline Fase 3 & 12 & 60 & & 23 & 115 & & - & - & & 41 & 205 & \\
\hline Fase 3+ & 10 & 50 & & 19 & 95 & & - & - & & 19 & 95 & \\
\hline Total & 38 & 190 & 9 & 58 & 290 & 16 & 69 & 345 & 11 & 100 & 500 & 10 \\
\hline
\end{tabular}

Nota. A participação de P3 foi encerrada na Fase 2.

Está representado na Tabela 2 somente o número total de mandos independentes emitidos pelos participantes. Em alguns casos, mesmo depois de alcançado o critério de conclusão da fase, mais sessões foram realizadas seguindo a orientação de autores (Bondy \& Frost, 1994; Ganz \& Simpson, 2004) segundo os quais os critérios de desempenho podem ser flexibilizados a fim de se garantir fluência do participante na prática da fase, expondo o indivíduo a um número maior de estímulos diferentes. Embora tenha se observado variabilidade entre o desempenho dos participantes três deles adquiriram as habilidades requeridas nas fases de ensino, alcançando os critérios de acertos. Todos os participantes apresentaram uma queda de desempenho após a inserção das figuras novas. Para cada novo estímulo introduzido, deveria ser alcançado o critério de $80 \%$ em três sessões consecutivas. Este critério foi responsável pelo número alto de sessões.

\section{Generalização}

As Figuras 1, 2 e 3 mostram o número de mandos emitidos por cada participante (P1, P2 e P4) na fase de generalização em cada sessão registrada. As cores das barras representam itens diferentes solicitados, exclusivos para cada participantes, incluindo preferidos e outros itens (neutros), tanto ensinados como não ensinados no PECS.

Generalização de P1. Na generalização na escola P1 apresentou um total de 107 mandos, por oito itens diferentes, todos previamente treinados. Os mandos foram sempre direcionados para sua professora, mesmo estando presentes os colegas e, algumas vezes, a auxiliar da escola. P1 apresentou generalização dos mandos tanto na presença quanto na ausência do item desejado. Do total de 107 respostas de mando, 43 foram por itens fora do campo de visão, itens necessários no contexto escolar e que não estavam disponíveis porque estavam guardados nos armários ou na mesa da professora. Os outros 64 mandos ocorreram na presença do item, quando os objetos estavam disponíveis na mesa ou na mão de um colega ou da professora.

Nos registros realizados na residência dos participantes foi observada generalização, embora em baixa frequência, de mandos por itens 
de preferência, comestíveis e não comestíveis. Por restrições da família de $\mathrm{P} 1$, a avaliação da generalização ocorreu apenas uma vez por semana. No total foram quatro registros e o número de mandos registrados de $\mathrm{P} 1$ foi menor em comparação aos demais participantes. Como para P1 foram quatro dias de coletas de dados de generalização, houve influência para um menor número de mandos registrados em comparação com os outros participantes. P1 apresentou oito mandos no contexto domiciliar, todos direcionados à mãe, sendo sete solicitações por itens não treinados. Somente um item estava fora da sua visão. A Figura 1 mostra o número de mandos emitidos e os itens solicitados por P1 na escola e na residência.

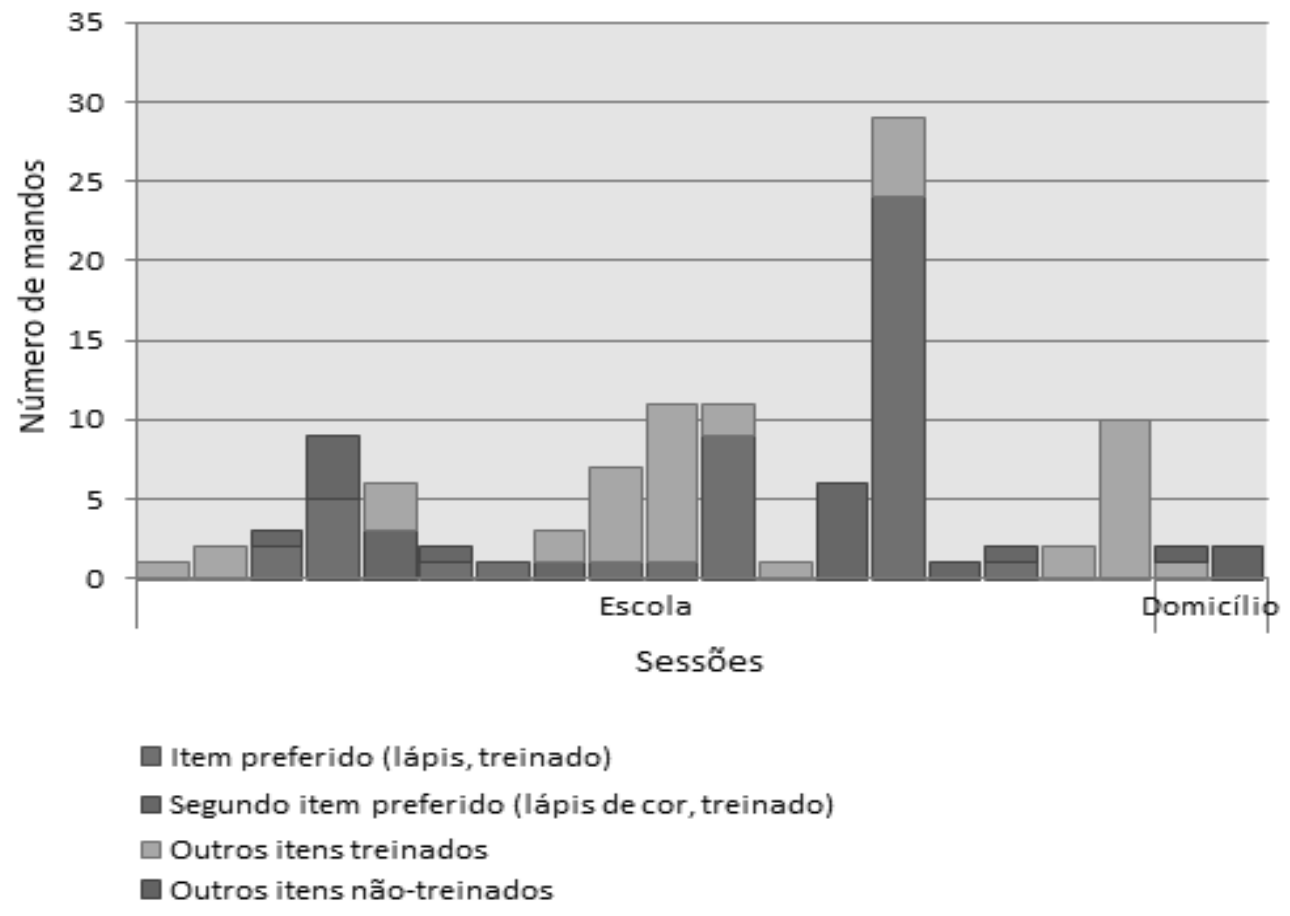

Figura 1. Número de mandos emitidos por P1 na fase de generalização.

Generalização de P2. P2 teve 20 sessões de registro de generalização na escola. $\mathrm{O}$ participante apresentou 118 mandos por nove itens, direcionados para sua professora, com uma exceção, quando foi direcionado a um colega. Todos os itens pedidos foram treinados. Os contextos foram a sala de aula e a quadra da escola, durante o recreio. No dia em que a sessão foi feita no recreio, a professora não registrou o número de mandos emitidos pela bola. P2 apresentou mandos generalizados tanto na presença quanto na ausência dos itens. Dos 118 mandos, 61 foram mandos por itens fora da visão, e os outros 57 mandos ocorreram na presença do item, quando os objetos estavam disponíveis na mesa ou nas mãos de um colega ou da professora. P2 apresentou mandos verbais esporadicamente, nomeando corretamente as figuras.
Em casa foram 21 registros em que P2 apresentou um total de 48 mandos por 13 itens diferentes, dos quais sete itens não haviam sido treinados. Os mandos em casa foram apresentados na presença do item (com exceção de uma emissão - para bombom) e direcionados para a mãe. Além de entregar a figura, P2 nomeou-as corretamente em todas as ocasiões no contexto domiciliar. Como a família de $\mathrm{P} 2$ não estava disponível para receber os pesquisadores para filmagens em casa uma vez por semana, a amostra de registros audiovisuais foi reduzida para a participante, sendo realizado duas vezes. A Figura 2 mostra o número de mandos emitidos e os itens solicitados por P2 na escola e na residência.

Generalização de P4. O teste de generalização de P4 na escola teve 17 sessões, feitas na 
hora do lanche, e foram utilizados somente itens comestíveis. Tais itens foram escolhidos porque P4 não possuía repertório para realizar atividades em sala de aula.

P4 apresentou 105 mandos por seis itens diferentes. Uma única vez foi pedido um item neutro não comestível (tesoura), o que parece ter sido uma escolha aleatória. Todos os itens estavam à vista do participante, fora do seu alcance. Todos os mandos foram direcionados à profes- sora e apenas um item solicitado, o bolinho, não havia sido treinado.

Em casa a coleta de P4 teve sete registros. P4 apresentou apenas 13 mandos, todos direcionados à mãe, para dois itens diferentes. Ficou alguns dias sem pedir nada, mas, segundo a mãe, não havia saciação pelos itens. Apenas dois mandos foram emitidos na ausência do item, e um item não havia sido treinado. A Figura 3 mostra o número de mandos emitidos e os itens solicitados por P4 na escola e na residência.

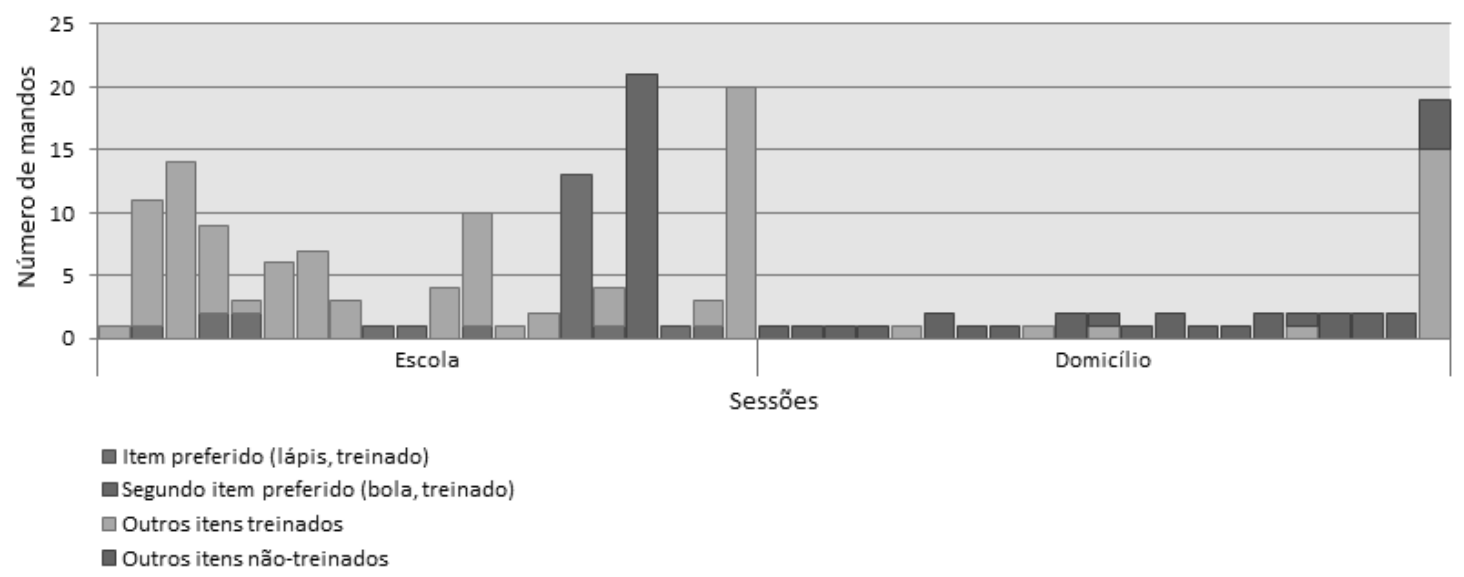

Figura 2. Número de mandos emitidos por P2 na fase de generalização.

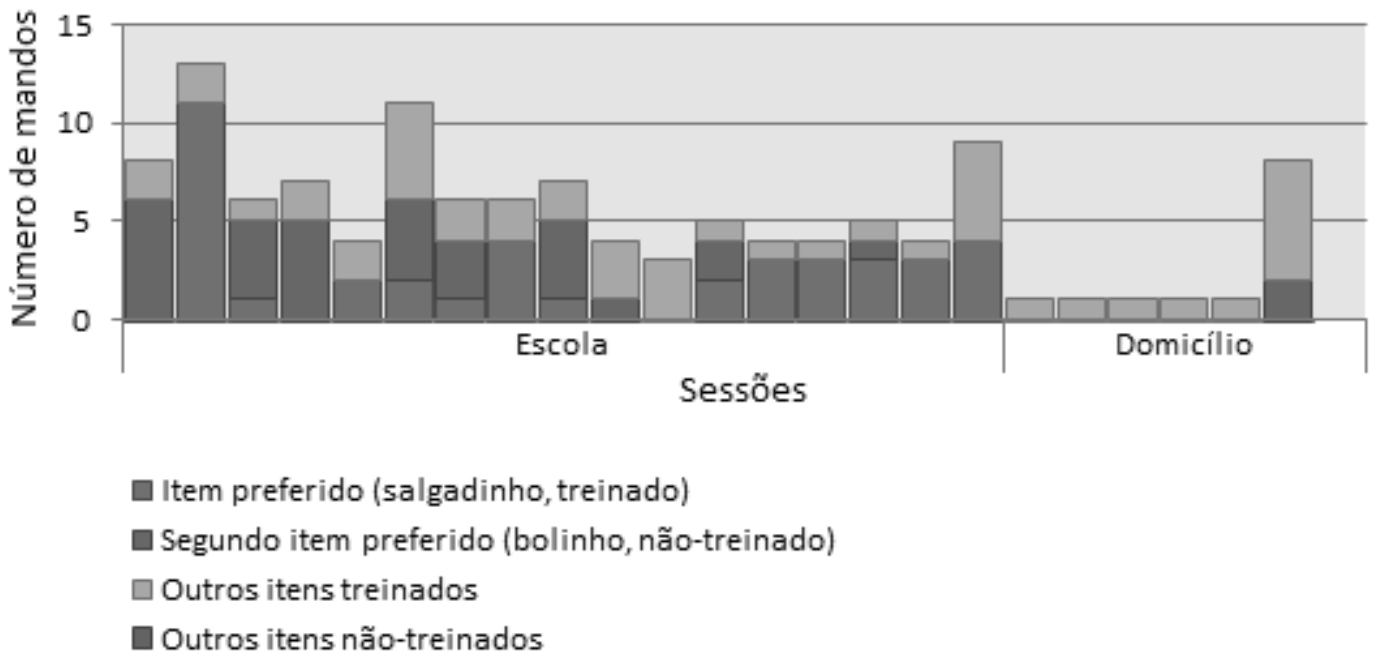

Figura 3. Número de mandos emitidos por P4 na fase de generalização.

\section{Discussão}

Os resultados da aquisição da comunicação por troca de figuras neste estudo permitem considerar que o PECS é eficaz para o ensino de mandos e para a verificação da generalização por autistas. Embora o treino e a generalização tenham sido bem sucedidos, algumas considerações que auxiliam a compreensão dos resultados merecem ser feitas. 
De acordo com Dogoe et al. (2010), variáveis como o repertório inicial do participante, diferenças individuais e as variáveis instrucionais do procedimento interferem na aquisição e na velocidade de ensino da comunicação. Frequentemente habilidades adquiridas pelos autistas em intervenções estruturadas não são generalizadas para o ambiente natural (Capellari, 2010; Klin et al., 2006). Para que a generalização ocorra, o ensino deve abarcar mudanças de ambientes e de parceiros de comunicação, treinando-se diretamente a resposta de interesse com vários instrutores e em múltiplos ambientes (Reeve, Reeve, Townsend, \& Poulson, 2007; Stokes \& Baer, 1977; Welsh, 2010).

Neste estudo, foi criada uma fase de treino complementar, a Fase 3+, que permitiu que os participantes entrassem em contato com itens necessários para o desempenho de atividades específicas ao ambiente (escola ou residência), o que pode ter contribuído para que o treino tenha se estabelecido de maneira suficientemente forte para facilitar a generalização.

Assim, os nossos resultados foram diferentes, em certa medida, dos encontrados em estudos prévios (Esbenshade \& Rosales-Ruiz, 2001; O’Neill \& Sweetland-Baker, 2001; Secan, Egel, \& Tilley, 1989), segundo os quais crianças autistas tiveram dificuldade em generalizar habilidades de comunicação para novas situações. Além da Fase 3+, planejada para maximizar a generalização, algumas outras variáveis do procedimento podem ter contribuído para os resultados na generalização, tais como a exposição dos participantes a variados estímulos na fase de treino e o uso de reforçadores de alta preferência pelos participantes.

Embora o número pequeno de participantes e a variabilidade do repertório de cada um deles possa dificultar a generalidade dos resultados para outros indivíduos, pode-se considerar que o delineamento de sujeito único compensa tal limitação uma vez que permite a análise do efeito do treino sobre o próprio desempenho de cada participante. Quanto aos ambientes, a Fase 3+, planejada para inserir novos parceiros de comunicação e novos contextos, ocorreu no mesmo contexto da escola em que foi avaliada posterior- mente a generalização, além dos mandos terem sido direcionados às mesmas pessoas. Em pesquisas futuras, ambientes diferentes devem ser incluídos na Fase 3, além de diferentes pessoas no contexto escolar com quem a criança possa se comunicar.

Quanto ao procedimento de ensino, uma limitação ao longo de todas as fases de treino relaciona-se ao critério de finalização. Os participantes, por algumas vezes, já teriam atingido o critério, mas o acréscimo de novas figuras os mantinha na mesma fase. Não pôde ser determinado se houve benefício com a exposição a uma variedade maior de estímulos, tanto para a aprendizagem quanto para a generalização, ou mesmo se a extensão das fases influenciou negativamente a motivação das crianças pelas atividades.

Futuros estudos poderiam tratar a generalização somente sob controle da operação motivacional, isto é, verificar a emissão de mandos sob controle de condição aversiva ou privação, como estar com sede ou pedir para usar o banheiro quando está na rua, por exemplo. Assim, as crianças emitiriam mandos na ausência do item na visão, por itens que necessitam, o que confere funcionalidade muito importante ao comportamento. Neste estudo foi planejado que a coleta deveria ser feita em ambiente minimamente modificado em relação à situação natural e não foi possível controlar a presença ou não do item. No ambiente familiar, a falta de condições de privação, por exemplo, podem explicar a baixa freqüencia de mandos quando comparada com a freqüência observada no ambiente escolar. Programar operações motivacionais no treino de mães e professores pode se constituir como passo importante no planejamento da generalização do PECS.

O estudo parece não ter criado condições especiais para promover, na escola, a interação do participante com outras pessoas além da professora. No entanto, futuros estudos poderiam utilizar o PECS, quando necessário, como ferramenta na promoção de inclusão social do autista na escola, desde que criem as condições especiais necessárias para promover, por meio de generalização planejada, maior interação do participante com outros alunos e funcionários. 


\section{Referências}

Ali, E., MacFarland, S. Z., \& Umbreit, J. (2011). Effectiveness of combining tangible symbols with the Picture Exchange Communication System to teach requesting skills to children with multiple disabilities including visual impairment. Education and Training in Autism and Developmental Disabilities, 46(3), 425-435.

American Psychiatric Association. (2013). Manual diagnóstico e estatístico de transtornos mentais (DSM-5) (5. ed.) Porto Alegre, RS: Artmed.

Bondy, A. S., \& Frost, L. A. (1994). The picture exchange communication system. Focus On Autistic Behavior, 9(3), 1-1.

Bosa, C., \& Schmidt, C. (2003). A investigação do impacto do autismo na família: Revisão crítica da literatura e proposta de um novo modelo. Interação em Psicologia, 7(2), 111-120.

Capellari, L. M. (2010). Educação e comunicação do autista e Asperger [Monografia Lato Sensu em Educação Especial]. São Paulo, SP: Centro de Referência em Distúrbios de Aprendizagem.

Carré, A. J., Le Grice, B., Blampied, N. M., \& Walker, D. (2009). Picture Exchange Communication (PECS) training for young children: Does training transfer at school and to home? Behaviour Change, 26, 54-65.

Charlop-Christy, M. H., Carpenter, M., Le, L., Leblanc, L. A., \& Kellet, K. (2002). Using the Picture Exchange Communication System (PECS) with children with autism: Assessment of PECS acquisition, speech, socialcommunicative behavior, and problem behavior. Journal of Applied Behavior Analysis, 35(3), 213-231.

Corsello, C. M. (2005). Early intervention in autism. Infants \& Young Children, 18(2), 74-85.

DeLeon, J. G., \& Iwata, B. A. (1996). Evaluation of a multiple-stimulus presentation format for assessing reinforcer preferences. Journal of Applied Behavior Analysis, 29(4), 519-533.

Dogoe, M. S., Banda, D. R., \& Lock, R. H. (2010). Acquisition and generalization of the Picture Exchange Communication System behaviors across settings, persons, and stimulus classes with three students with autism. Education and Training in Autism and Developmental Disabilities, 45(2), 216-229.
Esbenshade, P. H., \& Rosales-Ruiz, J. (2001). Programming common stimuli to promote generalized question-asking: A case demonstration in a child with autism. Journal of Positive Behavior Interventions, 3, 199-210.

Fazzio, D. F. (2002). Intervenção comportamental no autismo e deficiências de desenvolvimento: Uma análise dos repertórios propostos em manuais de treinamento (Dissertação de mestrado, Pontifícia Universidade Católica de São Paulo, SP, Brasil.)

Fidalgo, P. A., \& Godoi, P. J. (2008). Análise de um procedimento de comunicação funcional alternativa (Picture Exchange Communication System). Revista Brasileira de Terapia Comportamental e Cognitiva, 10(1), 51-66.

Fragale C., O’Reilly, M. F., Aguilar, J., Pierce N., Lang, R. B., Sigafoos, J., \& Lancioni, G. E. (2012). The influence of motivating operations on generalization probes of specific mands by children with autism. Journal of Applied Behavior Analysis, 45(3), 565-577.

Frost, L. A., \& Bondy, S. (1994). The Picture Exchange Communication System training manual. Cherry Hill, NJ: Pyramid Educational.

Gadia, C. A., Tuchman, R., \& Rotta, N. T. (2004). Autismo e doenças invasivas de desenvolvimento. Jornal de Pediatria, 80(2), 83-94.

Ganz, J. B., \& Simpson, R. L. (2004). Effects on communicative requesting and speech development of the Picture Exchange Communication System in children with characteristics of autism. Journal of Autism and Developmental Disorders, 34(4), 395-409.

Ganz, J. B., Simpson, R. L., \& Corbin-Newsome, J. (2008). The impact of the picture exchange communication system on requesting and speech development in preschoolers with autism spectrum disorders and similar characteristics. Research in Autism Spectrum Disorders, 2, 157 169.

Green, G. (2011). Análise do Comportamento aplicada ao autismo (D. Fazzio, Trad.). Recuperado em http://www.universoautista.com.br/autismo/ modules/works/item.php?id=5

Jurgens, A., Anderson, A., \& Moore, D. W. (2009). The effect of teaching PECS to a child with autism on verbal behavior, play, and social functioning. Behaviour Change, 26(1), 66-81. 
Klin, A., Chawarska, K., Rubin, E., \& Volkmar, F. (2006). Avaliação clínica de crianças com risco de autismo. Educação (Porto Alegre), 1(58), 255-297.

Klin, A., \& Mercadante, M. T. (2006). Autismo e transtornos invasivos do desenvolvimento. Revista Brasileira de Psiquiatria, 28(1), 1-26.

Lampreia, C. (2004). Os enfoques cognitivista e desenvolvimentista no autismo: Uma análise preliminar. Psicologia: Reflexão e Crítica, 17(1), 111-120.

Laraway, S., Snycerski, S., Michael, J., \& Poling, A. (2003). Motivating operations and terms to describe them: Some further refinements. Journal of Applied Behavior Analysis, 36(3), 407-414.

Lechago, S. A., Carr, J. A., Grow, L. L., Love, J. R., \& Almason, S. M. (2010). Mands for information generalize across establishing operation. Journal of Applied Behavior Analysis, 43(3), 381-395.

Llaneza, D. C., DeLuke, S. V., Batista, M., Crawley, J. N., Christodulu, K. V., \& Frye, C. A. (2010). Communication, interventions, and scientific advances in autism: A commentary. Physiology \& Behavior, 100, 268-276.

Martins, A. L. F. (2001). Avaliação dos distúrbios da linguagem no autismo infantil (Dissertação de mestrado, Universidade da Beira Interior, Covilhã, Portugal).

Murphy, C., Barnes-Holmes, D., \& Barnes-Holmes, Y. (2005). Derived manding in children with autism: Synthesizing skinner's verbal behavior with Relational Frame theory. Journal of Applied Behavior Analysis, 38(4), 445-462.

O’Neill, R. E., \& Sweetland-Baker, M. (2001). Brief report: An assessment of stimulus generalization and contingency effects in functional communication training in two students with autism. Journal of Autism and Developmental Disorders, 31, 235-240.

Reeve, S. A., Reeve, K. F., Townsend, D. B., \& Poulson, C. L. (2007). Establishing a generalized repertoire of helping behavior in children with autism. Journal of Applied Behavior Analysis, 40(1), 123-136.

Schopler, E., Reichler, R. J., \& Renner, B. (1988). The Childhood Autism Rating Scale (CARS). Los Angeles, CA: Western Psychological Services.
Secan, K. E., Egel, A. L., \& Tilley, C. S. (1989). Acquisition, generalization, and maintenance of question answering skills in autistic children. Journal of Applied Behavioral Analysis, 22, 181-196.

Skinner, B. F. (1957). Verbal behavior. New York: Appleton-Century-Crofts.

Souza, R. D. B., \& Juliani, J. (2012). O transtorno autista e a análise do comportamento. [Resumo]. In Centro Universitário Filadélfia (Ed.), $V$ Congresso de Psicologia da Unifil (pp. 59-64). Recuperado em http://www.unifil.br/portal/ images/pdf/documentos/anais/psicologia/vcongresso-de-psicologia.pdf

Stokes, T. F., \& Baer, D. M. (1977). An implicit technology of generalization. Journal of Applied Behavioral Analysis, 10(2), 349-367.

Sundberg, M. L., \& Partington, J. W. (1998). The assessment of basic language and learning skills. Pleasant Hill, CA: Behavior Analysts.

Tien, K. C. (2008). Effectiveness of the Picture Exchange Communication System as a functional communication intervention for individuals with autism spectrum disorders: A practice-based research synthesis. Education and Training in Developmental Disabilities, 43, 67-76.

Vollmer, T. R., Dozier, C. L., Jr., Borrero, J. C., Rapp, J. T., Bourret, J. C., \& Gadaire, D. (2007). Manipulating establishing operations to verify and establish stimulus control during mand training. Journal of Applied Behavior Analysis, 40(4), 645-658.

Welsh, A. (2010). The effects of picture exchange communication system training on the communication behaviors of young children with autism or severe language disabilities (Master thesis, Graduate School of the Ohio State University, Columbus, OH, USA). 